\title{
Normal-Sized Primary Lysosomes Are Present in Chediak-Higashi Syndrome Neutrophils
}

\author{
JAMES G. WHITE AND MARLYS KRUMWIEDE \\ University of Minnesota, Minneapolis, Minnesota 55455
}

\begin{abstract}
Azurophil granules of normal neutrophils are known to be primary lysosomes and contain myeloperoxidase activity. The present study has used ultrastructural cytochemistry to selectively stain myeloperoxidase containing granules of normal and Chediak-Higashi syndrome neutrophils and serial thin sections to determine if all peroxidase-positive organelles in Chediak-Higashi syndrome cells are protrusions of the huge inclusions characteristic of the disorder. Peroxidase-positive organelles in polymorphonuclear leukocytes from three patients with Chediak-Higashi syndrome varied in size from vesicles and normal-sized lysosomes to the huge bodies filling the cytoplasm. Serial sections demonstrated that the small and normal-sized organelles were commonly present and independent of the giant granules. One type of the normalsized lysosomes contained small vesicles free of peroxidase activity, conveying a honeycomb-like appearance to the matrix. This variety, in particular, was identified in various stages of fusion with giant organelles in circulating Chediak-Higashi syndrome neutrophils. (Pediatr Res 22: 208$215,1987)$
\end{abstract}

\section{Abbreviations}

CHS, Chediak-Higashi syndrome

PMN, polymorphonuclear leukocyte

$\mathrm{H}_{2} \mathrm{O}_{2}$, hydrogen peroxide

HBSS, Hanks' balanced salt solution

CCD, citrate-citric acid-dextrose

Giant organelles present in the cytoplasm of circulating granulocytes support the diagnosis of CHS (1-4). The massive leukocyte inclusions have been subjected to many biochemical, histochemical, cytochemical, and immunochemical procedures and explored in detail by light, phase contrast, and electron microscopy to define their nature (5-9). Because patients with CHS are particularly susceptible to bacterial infection, most studies have focused on the PMN (10-15). Investigations of neutrophil precursors in bone marrow from patients and animal models suggested that azurophil lysosomes developing in promyelocytes undergo fusion during maturation to form the giant inclusions observed in peripheral blood cells (1-9). Various investigators concluded that the process of fusion was exclusively concerned with the azurophilic organelles of promyelocytes and myelocytes, that it was complete before the cells entered peripheral blood, and that nonlysosomal organelles, called specific or

Received September 12, 1986; accepted March 27, 1987

Correspondence James G. White, M.D., University of Minnesota Health Sciences, Departments of Pediatrics, Laboratory Medicine and Pathology, UMHC Box 490, Harvard at East River Road, Minneapolis, MN 55455.

Supported by USPHS Grants HL-11880, CA-21737, GM-22167 and a Grant MOD 1-886 from the March of Dimes. secondary granules, were not involved in the evolution of giant inclusions $(5,15,16)$.

Early findings in our laboratory (18-21) and subsequent findings of Rausch et al. (22) disagreed with the prevailing concept. Although azurophil granules were clearly involved in formation of the large inclusions, the process of incorporation did not appear to be completed before departure from bone marrow. Small, normal-sized azurophil lysosomes were evident in circulating PMN, and sites of fusion between some of them and huge neutrophil inclusions were defined (18-21). Occasional specific granules, negative for the reaction product of acid phosphatase or myeloperoxidase, were also identified, along with small zones of trapped cytoplasm, in the huge inclusions $(21,22)$. Combined with the evidence that many giant PMN organelles were in various stages of internal disintegration and the presence of other abnormal features in the cytoplasm of circulating PMN, we concluded that CHS was a lysosomal disease and that many of the huge organelles were secondary, rather than primary, lysosomes.

Recently, these observations have been seriously challenged (23). West (23) has carried out a biochemical study by first lysing the neutrophils from two brothers with CHS and then isolating the cytoplasmic organelles by density gradient centrifugation. Only normal specific granules and giant lysosomes were found. Two different azurophil granules, normally present and separable, were absent from patient cells. Light microscopy of Wright stained blood smears from the two CHS patients provided convincing support for the absence of normal-sized azurophilic granules in their PMN. Based on these findings, West (23) concluded that normal-sized lysosomes described in thin sections of CHS neutrophils were, in reality, convolutions or protrusions of giant granules just outside the plane of section.

The present investigation has examined serial thin sections of neutrophils from three unrelated patients with CHS stained for myeloperoxidase activity. Results demonstrate a wide variation in the size of myeloperoxidase-positive organelles in CHS neutrophils, ranging from small vesicles to normal-sized lysosomes and to the huge inclusions characteristics of the disease.

\section{MATERIALS AND METHODS}

Patients. Neutrophils from the blood samples of 11 patients with CHS have been evaluated in our previous ultrastructural studies. For the present investigation blood was obtained from three unrelated patients with CHS. One patient is a young adult male. Clinical features of his disease have been described previously (18-21). The others are a female infant and a young boy with laboratory features typical of CHS. Features of their blood cells on peripheral smears were identical to each other and to the other patients with CHS whom we have evaluated.

White blood cell preparations. Blood was drawn from normal controls and patients with CHS and mixed immediately with CCD (sodium citrate, $93 \mathrm{mmol} /$ liter; citric acid, $70 \mathrm{mmol} / \mathrm{liter}$; and dextrose, $140 \mathrm{mmol} / \mathrm{liter} ; \mathrm{pH} 6.5 ; \mathrm{CCD}$ ) in a ratio of 9 parts 
blood to 1 part anticoagulant. The blood samples were sedimented at room temperature for 20 min under a force of $200 \times$ $g$. Buffy coats were either fixed in place or aspirated and transferred to separate tubes for fixation. For cytochemical studies the blood was collected in heparinized syringes with $6 \%$ dextran in saline (Dextran 75, Travenol) and the syringes were inverted and allowed to sediment spontaneously at room temperature $(24,25)$. The leukocyte-rich plasma from buffy coats or spontaneously sedimented samples were washed in HBSS or sedimented in Ficoll-Hypaque (Pharmacia and Winthrop) to obtain nearly pure granulocyte preparations.

Preparation for electron microscopy. Leukocyte suspensions from control individuals and CHS patients were combined with an equal volume of $0.1 \%$ glutaraldehyde in White's saline [a $10 \%$ solution of a 1:1 mixture of $\mathrm{A}: 2.4 \mathrm{M} \mathrm{NaCl}, 0.1 \mathrm{mM} \mathrm{KCl}$, $0.1 \mathrm{mM} \mathrm{KCl}, 46 \mathrm{mM} \mathrm{MgSO}_{4}, 64 \mathrm{mM} \mathrm{Ca}\left(\mathrm{NO}_{3}\right)_{2} \cdot 4 \mathrm{H}_{2} \mathrm{O}$ and $\mathrm{B}$ : $0.13 \mathrm{M} \mathrm{NaHCO}_{3}, 8.4 \mathrm{mM} \mathrm{NaH}_{2} \mathrm{PO}_{4}$, and $0.1 \mathrm{~g} / \mathrm{L}$ of phenol red, $\mathrm{pH}$ 7.4] (26). After $15 \mathrm{~min}$ the cell suspensions were centrifuged and the supernatant was replaced with $3 \%$ glutaraldehyde in the same buffer. The cells were resuspended in this fixative for 30 $\min$ at $4^{\circ} \mathrm{C}$. Cell samples were then centrifuged to buttons, and the aldehyde fixative removed and replaced by $1 \%$ osmium tetroxide with $1.5 \%$ potassium ferrocyanide $(21,27)$. Some of the aldehyde-fixed leukocyte samples were combined with glycerol, frozen in liquid nitrogen, and fractured under high vacuum. Carbon-platinum replicas of the fractured cells were cleaned in detergent before examination (20).

Cytochemistry. Cytochemical studies were carried out on leukocyte samples fixed in $2.5 \%$ paraformaldehyde and $0.5 \%$ glutaraldehyde in $0.1 \%$ cacodylate buffer. Myeloperoxidase activity was evaluated by the method of Graham and Karnovsky (28) as modified in our laboratory (29). After initial fixation in paraformaldehyde-glutaraldehyde the cell samples were washed several times in buffer, incubated for $1 \mathrm{~h}$ in medium containing diaminobenzidine, hydrogen peroxide and dimethylsulfoxide, and postfixed in osmic acid. Hydrogen peroxide or diaminobenzydine was left out of the incubation system in some experiments. In others the inhibitor, aminotriazole, was added to the incubation mixture.

All samples were washed in buffer after postfixation in osmic acid, dehydrated in a graded series of alcohols, and embedded in Epon 812. Thin sections of samples for morphology were stained with uranyl acetate and lead cytrate to enhance contrast. Sections from samples incubated for peroxidase activity were examined unstained. Serial sections prepared from the peroxidase-stained material were mounted on slotted grids specially made for this purpose. All observations were made in a Philips 301 electron microscope.

\section{RESULTS}

General. Ultrastructural and cytochemical features of neutrophils from patients with CHS have been described in detail in previous investigations (18-21). Giant cytoplasmic granules are the characteristic feature of the disorder. The hugh organelles manifest a wide variety in size, shape, electron density, internal content, and substructural organization (Fig. 1). Some of the large bodies are spherical in form and display a homogenous internal structure. All of the organelles in CHS eosinophils are of this type (Fig. 2). Most of the massive structures in CHS neutrophils, however, are very irregular. Areas of contact and fusion of the huge bodies with one another and with both large and small organelles in the adjacent cytoplasm were identified in previous investigations (21), as well as in cytochemically stained thin sections and replicas of freeze-fractured CHS neutrophils in the present study (Figs. 3 and 4). Selective staining for myeloperoxidase, an enzyme localized to azurophil primary lysosomes, confirmed the suggestion that a process of fusion was a continuing process in the circulating neutrophil. Giant irregular inclusions were in contact and in various stages of fusion with normal-sized, myeloperoxidase-positive granules and segments of cytoplasm within the evolving massive structures (Fig. 6). These observations have been consistent in neutrophils from all 11 of the CHS patients we have studied.

Normal-sized primary lysosomes in CHS neutrophil. In the present study slotted grids (SPI Supplies, Westchester, PA) have been employed to permit photographic evaluation of five to 12 consecutive thin sections of the same cell. Samples of neutrophils from three unrelated patients were used in order to provide a reasonable spectrum of CHS in man. Neutrophil samples had been selectively stained for myeloperoxidase activity with a procedure extensively used for this purpose (21). Controls studied in which $\mathrm{H}_{2} \mathrm{O}_{2}$ or diaminobenzidine were left out of the incubation mixture or the cells incubated with aminotriazole before incubation revealed absence of organelle staining.

Reaction product of myeloperoxidase activity was confined to azurophilic lysosomes of control neutrophils. The primary granules containing the enzyme activity were randomly distributed, oval to spherical, and uniform in size and electron density. Unstained secondary granules, slightly smaller and more elongated, filled the cytoplasmic area not occupied by primary lysosomes.

Thin sections of PMN from patients with $\mathrm{CHS}$ revealed a strikingly different distribution of myeloperoxidase reaction product (Fig. 5). Particles containing electron-dense osmium black ranged in size from small vesicles to huge inclusions which nearly filled the cytoplasm of the cell. The size distribution, however, was not uniform. Smaller granules positive for myeloperoxidase ranged from the size of vesicles upward to the size of normal azurophil lysosomes (Fig. 7). The next group stained by reaction product were relatively spherical, uniformly dense giant granules (Fig. 8). These organelles were often less intensively stained by osmium black than the small lysosomes and hugh irregular inclusions (Fig. 9). The latter structures were the dominant organelles in mature CHS neutrophils. Their irregular form often brought them close to the small, normal-sized lysosomes and many points of fusion between them could be identified (Fig. 9A).

Serial sections, however, indicate clearly that many of the normal-sized lysosomes and smaller organelles stained for myeloperoxidase are not attached or incorporated into giant CHS inclusions (Figs. 7-9). Small lysosomes were evident in almost every thin section through a CHS neutrophil and often outnumbered the giant granules. The small organelles were frequently passed through in three to four sections (Fig. 7). Normalsized lysosomes were sliced from one side to the other in four to six sections. Giant lysosomes were rarely sectioned completely in the eight to 12 consecutive sections picked up on a single grid (Fig. 8).

The small, peroxidase-positive granules were uniformly stained by reaction product (Fig. 7). Slightly larger lysosomes contained regularly arranged, electron-transparent areas, producing a honeycomb appearance. Many of these structures were cut completely in six to eight serial sections. Their involvement in fusion with giant lysosomes was easily identified with (Fig. $8 B$ ) or without (Fig. 3) staining for peroxidase.

Another type of lysosome was also cut completely in serial sections. Ring-shaped lysosomes are the characteristic form found in CHS monocytes and observed occasionally in eosinophils and neutrophils (Fig. $8 A$ ).

Sectioning completely through such structures suggests that they are membrane enclosed spheres.

\section{DISCUSSION}

The present investigation has employed ultrastructural cytochemistry and serial sectioning to determine whether CHS neutrophils contain normal-sized primary lysosomes. Giant, peroxidase-positive granules are the characteristic cytologic feature of CHS. At least two distinct varieties have been distinguished in 


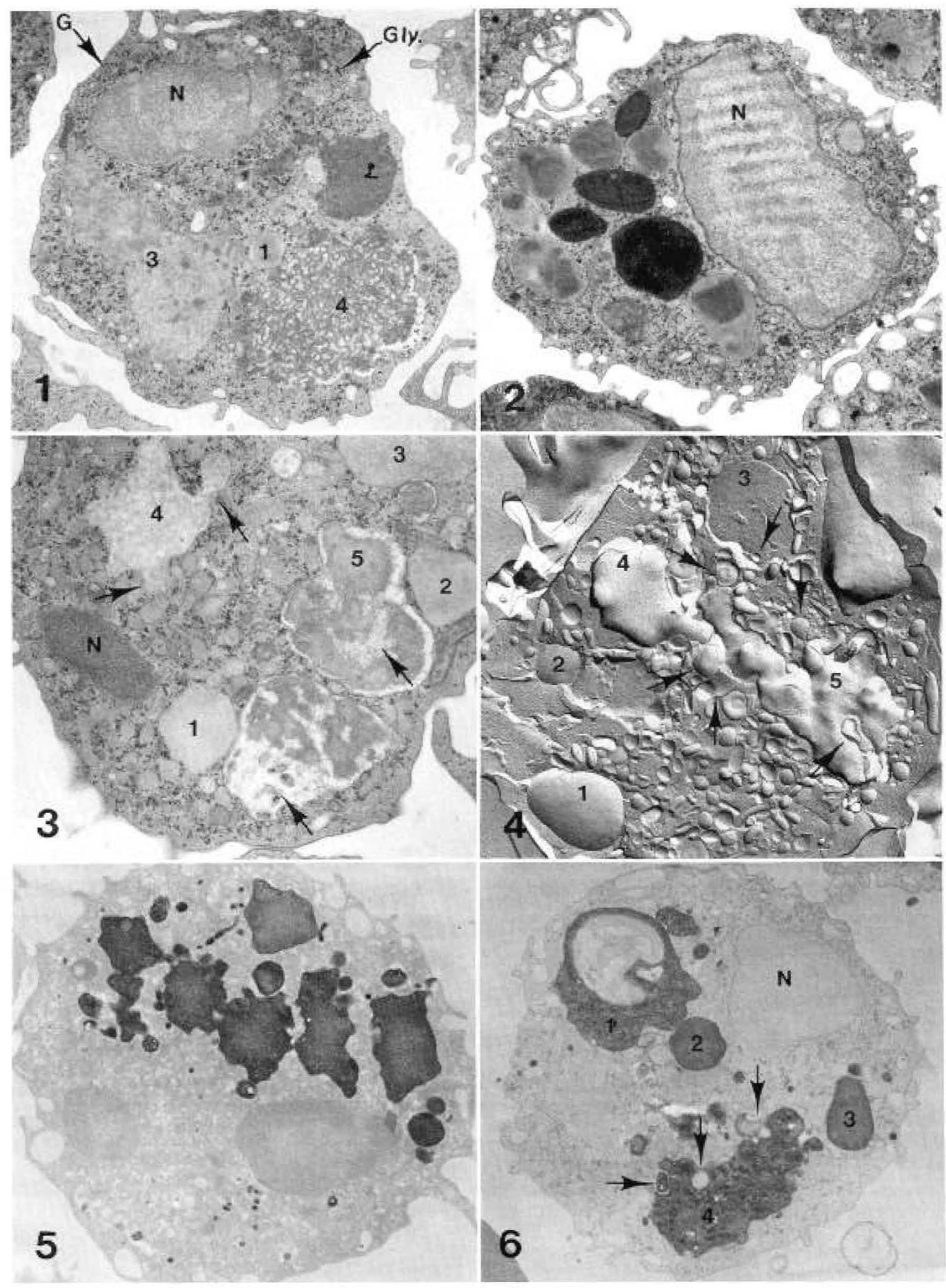

Fig. 1. CHS neutrophil. Thin section of a PMN from a sample of leukocytes fixed in glutaraldehyde and osmic acid containing potassium ferrocyanide. The cell contains a portion of the nucleus $(N)$, numerous glycogen particles $(G l y)$, and an abundance of normal-sized granules $(G)$ and several giant organelles. One ( $l$ ) of the CHS organelles is small, compared to the others and spherical in form. Another (2) is irregular and relatively electron dense. A third (3) is very large and fills a considerable area of the cytoplasm. The fourth (4) is almost as large, but radically different in substructure. Its matrix appears made up of a complex array of membranes not apparent in any of the other giant granules in this cell. $\times 7800$.

Fig. 2. CHS eosinophil. The giant lysosomes of CHS eosinophils are hugh, but resemble normal size eosinophil granules in most respects. Some are irregular in shape, but most are round or oval. Surface contours are smooth and the substructure is like that of granules in normal cells, except for the presence of several crystals rather than one. Giant organelles filled with debris of the type common in CHS neutrophils (lysosome 4 in Figure 1) are not found in patient eosinophils. $\times 7800$. 
morphological, cytochemical, and physiological studies of circulating PMN (18-21). One variety is round or oval with smooth contours and a homogenous internal structure. It resembles normal-sized azurophil lysosomes in form and substructure, but is many times larger. The second type of huge organelle is extremely irregular and usually much larger than the first. Basophil and eosinophil granulocytes from CHS do not contain giant lysosomes of the second type found in neutrophils, and the irregular structure is much less common in promyelocytes, myelocytes, and young PMN in the bone marrow, compared to circulating leukocytes.

The first type of giant granule in CHS neutrophil forms as a result of fusion in the promyelocyte and myelocyte and has reached what appears to be a stable configuration before release of the cell from the marrow (15-17). Huge irregular inclusions with many protuberances may have developed in the same manner as smoothly contoured giant granules, but continue to grow at the expense of other large bodies and normal-sized lysosomes, even in the circulating neutrophil (21). Points of contact and fusion were identified in previous publications, but are even more clearly demonstrated in the serial sections evaluated in the present study. In addition to fusion with large and small lysosomes the huge irregular inclusions have been shown to incorporate secondary granules and fragments of cytoplasm into their substructure $(18,19,21,22)$. Serial sections also demonstrate this phenomenon well, and suggest that incorporation is the result of trapping within tortuous interstices of the irregular inclusions as they fuse with other lysosomes. As a result of the continuing interaction with other organelles and components of cytoplasm the huge irregular bodies develop marked alterations of their internal organization so that they often resemble bags of debris $(18,19)$. The changes are consistent with the suggestion that many of the huge irregular inclusions in CHS neutrophils are secondary, rather than primary lysosomes (21, $30,31)$.

The recent investigation reported by West (23) provided strong arguments against the hypothesis proposed above, and prompted the current study. In his investigation neutrophils from two brothers with CHS were isolated, suspended in heparinized sucrose, lysed, and filtered. The granule-rich filtrate was centrifuged on a continuous sucrose gradient, and fractions were assayed for protein and enzyme activities. Bands isolated from CHS neutrophils differed from those obtained from normal cells. CHS gradients contained a single broad band representative of primary lysosomes and completely lacked the two bands characteristic of the two varieties of azurophil granules in normal PMN. A fraction identical to the secondary granules from normal neutrophils was obtained from CHS gradients. Based on these findings and biochemical analyses of the gradient fractions, West concluded that there was no evidence for the presence of normal azurophilic granules of any kind in the CHS neutrophil from peripheral blood. In order to confirm his observations West studied Wright stained blood smears from the two brothers with CHS. Only giant granules were evident in neutrophils from the two patients, although their color was neither azurophilic or basophilic. Thus, blue-gray or slate-gray organelles were present in CHS neutrophils, but there were no azurophilic granules of any kind in the cells.

The present study and previous reports have used the terms azurophil granule or lysosome, primary granule or lysosome, peroxidase-positive granule or lysosome and giant CHS granules, lysosomes, bodies, or inclusions interchangeably (1-22, 30, 31). As indicated by West (23) “... precedent favors continued use of 'azurophilic' to describe them, if not their color, because it has become synonymous with 'peroxidase-positive' which they are." We agree that the avidity for Romanowsky dyes is not as important a distinguishing feature of the primary lysosome as its reaction to staining for myeloperoxidase.

Serial sections evaluated in the present study demonstrate that the giant granules and inclusions in CHS neutrophils are peroxidase-positive. They also reveal that organelles similar in size to primary lysosomes, or smaller, are present in the patient's PMN and also react intensely for peroxidase enzyme. Staining of the organelles was blocked by absence of $\mathrm{H}_{2} \mathrm{O}_{2}$ or diaminobenzidine from the incubation mixture, or the presence of the inhibitor, aminotriazole (21). The small, peroxidase-positive organelles were independent of the giant CHS organelles and, therefore, fulfill the definition of either primary lysosomes or azurophilic granules as West (23) has suggested.

It is uncertain why the CHS neutrophils studied by West appeared to lack normal-sized azurophilic granules or primary lysosomes. He considered the possibility that his observations might be due to genetic variability of the disease, since the patients he studied were brothers. However, normal-sized primary granules stained for myeloperoxidase activity have been observed in neutrophils from all CHS patients in whom we have carried out the reaction. Serial sections of PMN from the three patients examined in this study show clearly that most of the small azurophilic lysosomes are not protuberances of giant granules, but independent structures. Their presence in circulating PMN does not mean they are "normal" primary lysosomes; continued interaction and fusion with giant granules suggest they are not $(18-22,30-32)$. The critical point is that they are similar in size or smaller than primary lysosomes in normal PMN. Their existence and the presence of more than one variety of giant lysosome in circulating PMN, but not in other granulocytes (31,

Fig. 3. Portion of a neutrophil from a patient with CHS. Three giant lysosomes in the cytoplasm $(1,2,3)$ have relatively smooth contours and a homogenous substructure. One of them (3) appears in close contact with a smaller granule and may be in the process of fusion with it. Another (4) giant lysosome is irregular and appears to have fused with at least two smaller organelles $\left(_{\uparrow}\right)$. Two other huge bodies $(5,6)$ have a very complicated internal organization. One of them (5) contains parallel filaments $\left(_{\uparrow}\right)$ running length wise and in cross-section, resembling those frequently observed in neutrophil cytoplasm. The other (6) appears to have taken up fluid, resulting in dilution of the matrix. Fragments of cytoplasm ( $\uparrow)$ are apparent inside its limiting membrane. $\times 9900$.

Fig. 4. Replica of freeze-fractured CHS neutrophil. Several giant organelles are evident in the cytoplasm of this cell. Two $(1,2)$ have smooth surfaces. Another (3) has been fractured through and contains a relatively smooth matrix. However, a membrane profile similar in size to that of normal-sized cytoplasmic granules is apparent just inside its limiting membrane $(\uparrow)$. Two giant organelles $(4,5)$ have tortuous surface contours. Areas of contact and possible fusion with several smaller organelles can be identified $\left({ }_{\uparrow}\right)$. The marked irregularity of this variety of CHS organelle may be due to continued interaction with smaller granules. $\times 9900$.

Fig. 5. CHS neutrophil from sample of leukocytes incubated for peroxidase activity. Reaction product is localized to lysosomes of variable size and surface configuration. Those in the upper half of the figure are mainly giant lysosomes. A number of normal-sized granules stained for peroxidase activity are evident among the large organelles, and also separate from them in the lower half of the figure. $\times 9900$.

Fig. 6. CHS neutrophil incubated for myeloperoxidase activity. The variability in size and complexity of CHS organelles is further indicated by this example. One of the huge bodies $(1)$ appears wrapped around an area of cytoplasm. Two $(2,3)$ have smooth contours, while another $(4)$ is irregular and appears in the process of fusion with and incorporation of smaller organelles $(\uparrow) . \times 9900$. 

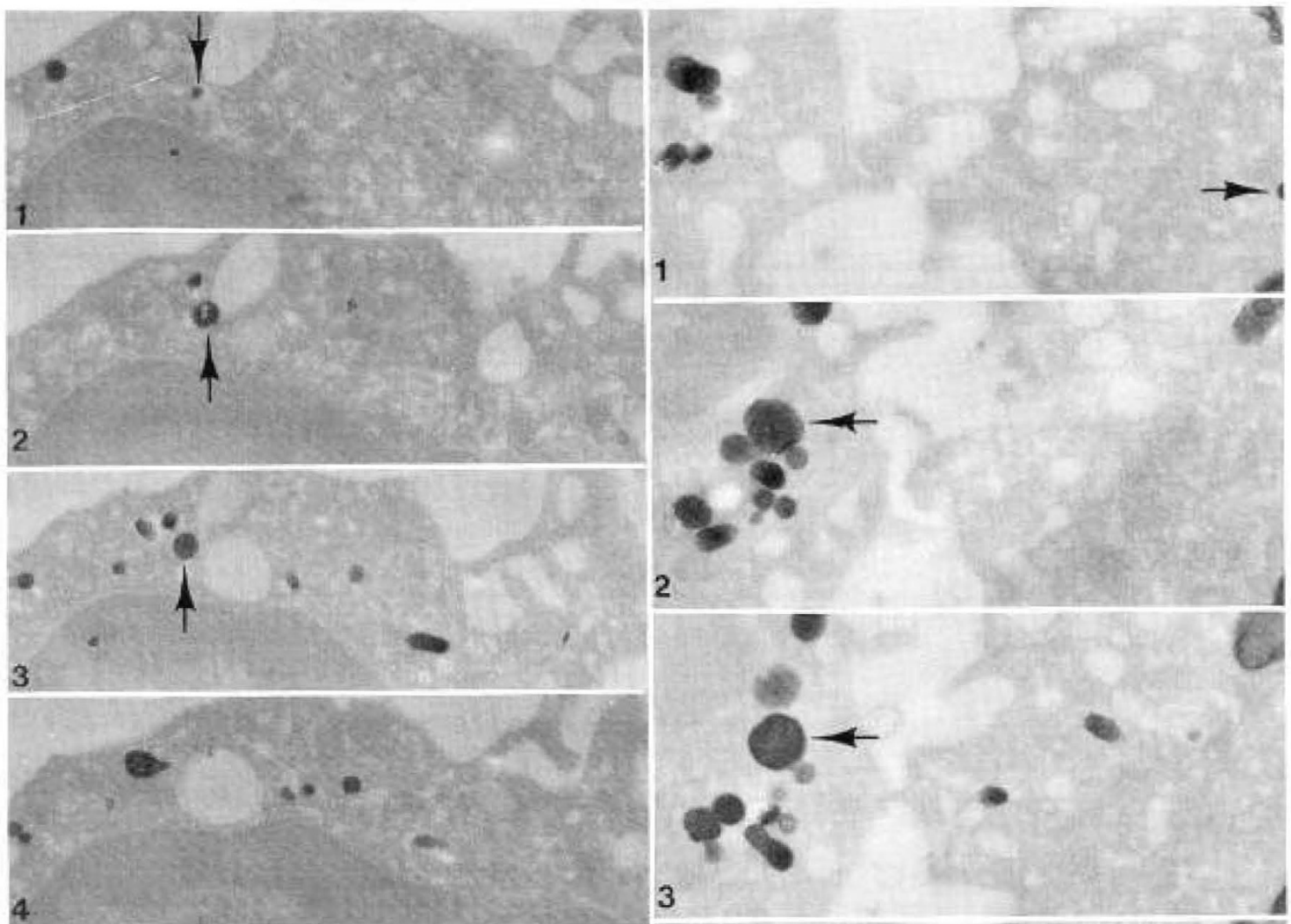

3
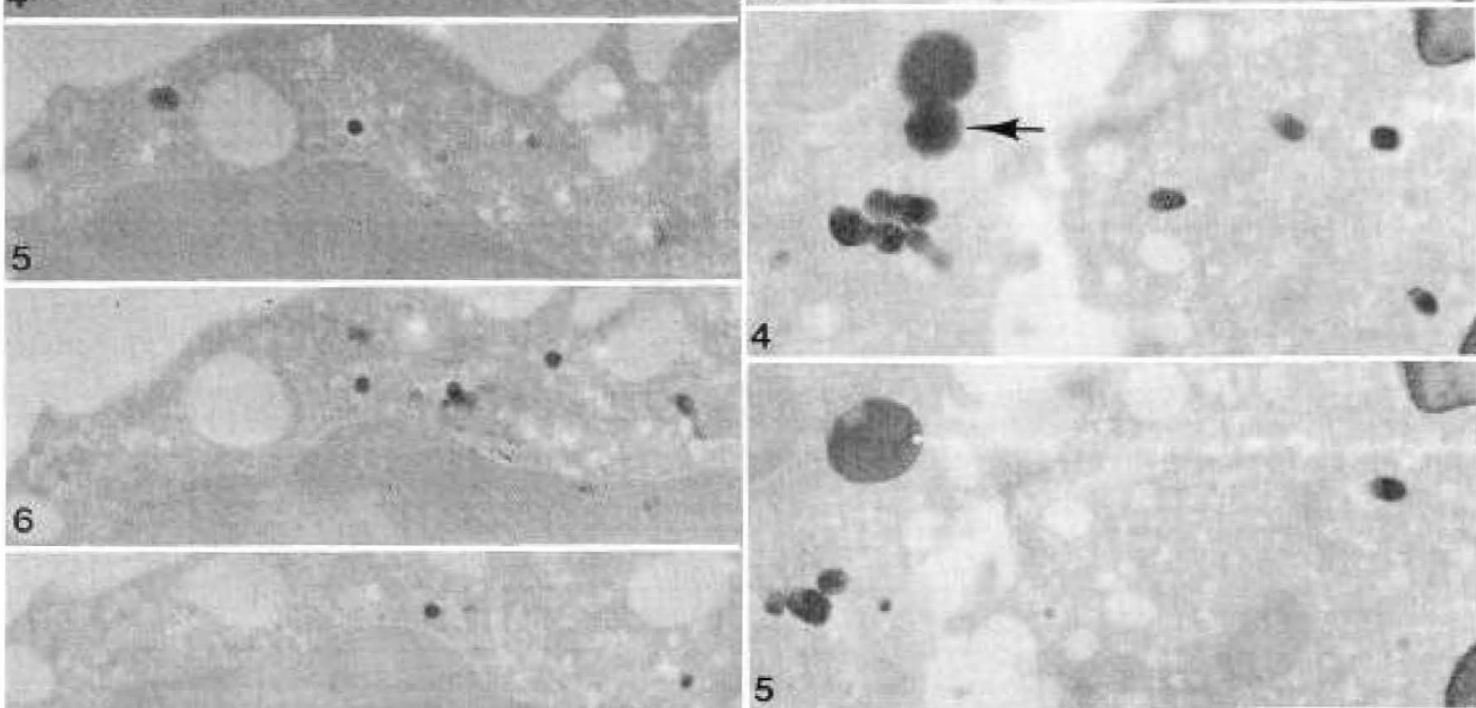

6

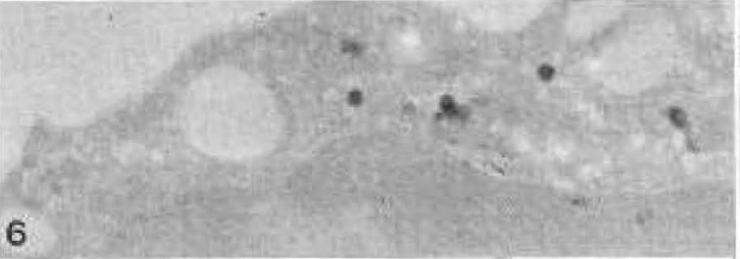

4

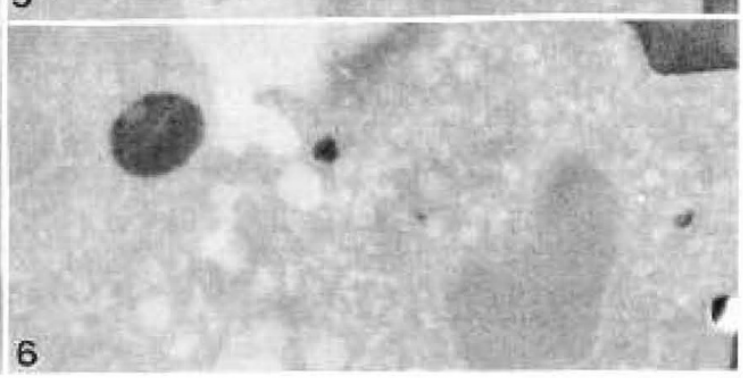

8

7

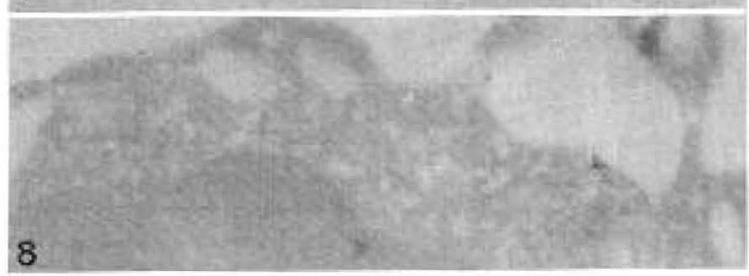

7A

7B

Fig. 7. A, (1-8) serial sections through an area of CHS neutrophil cytoplasm stained for myeloperoxidase activity. A normal-sized granule containing reaction product in the first section (1) disappears in the second (2). A barely perceptible organelle $(\uparrow)$ in section 1 becomes prominent in section $2(\uparrow)$, along with two others. The prominent lysosome remains in section 3, but disappears in section 4 . New peroxidase-positive granules appear in section 3, 4, and 5, but are gone in one or two sections. By section 8 the area of cytoplasm is free of lysosomes. At least 14 normal-sized granules selectively stained by reaction product were completely transected in the eight sections. All $\times 14,000$. B, serial sections through areas of

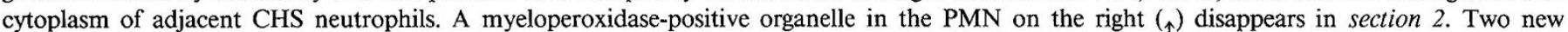
organelles appear in section 3 and two more in section 4. Three of these areas are gone in section 5 and the other has disappeared in section 6 as new organelles appear. The small group of peroxidase-positive lysosomes in the cell on the left increases from four in section 1 to eight in section 2 . A large lysosome $\left(_{\uparrow}\right)$ appearing in section 2 remains prominent in sections 3 and 4 , but is replaced by an even larger peroxidase-positive organelle appearing in section 4 and continuing through section 6 . The group of small lysosomes in the cell on the left in section 2 remains closely associated, but disappears completely in section 6 . All $\times 16,500$. 
32 ), suggest that the process of fusion leading to giant granule formation is not complete before the cells leave the bone marrow. However, it is also possible that the process of fusion leading to giant granules was complete and that the small and normal-sized primary lysosomes present in circulating neutrophils resulted from fission or budding off from the enlarged organelles. Fusion appears to be a common, virtually continuous process in CHS neutrophils, but it is not always easy to distinguish fusion from fission in the electron microscope.

Note added in proof. Since submission of this report, the authors have examined the ultrastructure and cytochemistry of PMN leukocytes from one of the brothers studied by Dr. West through the courtesy of Dr. Jo White and Dr. John Gallin of the National Institutes of Health. Small and normal sized, peroxidase-positive lysosomes, as well as densely stained, giant inclusions identical to those described in PMN from three patients in the present study, were identified in his neutrophils.

\section{REFERENCES}

1. Beguez-Cesar A 1943 Neutropenia cronica maligna familiar con granulacione atipicas de los leucocitos. Soc Cubana Pediatr Bol 15:900-922

2. Steinbrinck W 1948 Uber eine neue Granulationsanomalie der Leukocyten Dtsch Arch Klin Med 193:577-581

3. Chediak MM 1952 Nouvelle anomalie Leucoytaire de caractere constitutionnel et familial. Rev Hematol 7:362-367

4. Higashi O 1954 Congenital gigantism of peroxidase granules: The first case ever reported of qualitative abnormality of peroxidase. Tohuku J Exp Med 59:315-322

5. Bernard J, Bessis M, Seligmann M, Chassigneux J, Chome J 1960 Un case de maladi de Chediak-Steinbrinck-Higashi. Etude Clin Cytol 68:563-566

6. Astaldi G, Strosselli E 1961 Histochimie des granulations geantes des polynucleaires dans l'anomalie de Chediak. Nouv Rev Fr Hematol 1:626-628

7. Bessis M, Bernard J, Seligmann M 1961 Etude cytologique d'un cas de maladie de Chediak. Nouv Rev Fr Hematol 1:422-440

8. Sadan N, Yaffe D, Rozenszajn L, Adar H, Soroker B, Efrati P 1965 Cytochemical and genetic studies in four cases of Chediak-Higashi-Steinbrinck syndrome. Acta Haematol 34:20-29

9. Wolff SM, Dale DC, Clark RA, Root RK, Kimball HR 1972 The Chediak Higashi syndrome: studies of host defenses. Ann Intern Med 76:293-306

10. Blume RS, Wolff SM 1972 The Chediak-Higashi syndrome: studies in four patients and a review of the literature. Medicine 51:247-280

11. Klebanoff SJ, Clark RA 1978 Function and clinical disorders. North-Holland Publishing Co, New York, pp 735-792

12. Clawson CC, Repine JE, White JG 1971 Chediak-Higashi syndrome: quantitative defect in bactericidal capacity. Blood 38:814
13. Clark RA, Kimball HR, Padgett GA 1972 Phagocytosis in chronic granulomatous disease and the Chediak-Higashi syndrome. Blood 39:644-649

14. Stossel TP, Root RK, Vaughan M 1972 Phagocytosis in chronic granulomatous disease and the Chediak-Higashi syndrome. N Engl J Med 286:120-123

15. Root RK, Rosenthal AS, Balestera DJ 1972 Abnormal bactericidal, metabolic and lysosomal functions of Chediak-Higashi syndrome leukocytes. J Clin Invest 51:649-665

16. Davis WC, Spicer SS, Greene WB, Padgett GA 1971 Ultrastructure of bone marrow granulocytes in normal mink and mink with the homolog of the Chediak-Higashi trait of humans: I. Origin of the abnormal granules present in the neutrophils of mink with the CHS trait. Lab Invest 24:303-317

17. Davis WC, Spicer SS, Greene WB, Padgett GA 1971 Ultrastructure of cells in bone marrow and peripheral blood of normal mink and mink with the homologue of the Chediak-Higashi trait of humans: II. Cytoplastic granules in eosinophils, basophils, mononuclear cells and platelets. Am J Pathol 63:411-432

18. White JG 1966 The Chediak-Higashi syndrome: a possible lysosomal disease. Blood 28:143-156

19. White JG: The Chediak-Higashi syndrome: cytoplasmic sequestration in circulating leukocytes. Blood 29:435-451

20. White JG 1973 The Chediak-Higashi syndrome: fine structure of giant inclusions in freeze-fractured neutrophils. Am J Pathol 72:503-520

21. White JG, Clawson CC 1980 The Chediak-Higashi syndrome: the nature of the giant neutrophil granules and their interactions with cytoplasm and foreign particulates. Am J Pathol 98:151-196

22. Rausch PG, Pryzwansky KB, Spitznagel JK 1978 Immunocytochemical identification as azurophilic and specific granule markers in the giant granules of Chediak-Higashi neutrophils. N Engl J Med 298:693-698

23. West BC 1986 Chediak-Higashi syndrome neutrophils are characterized by the absence of both normal azurophilic granules. Am J Pathol 122:177-189

24. White JG 1972 Giant organelles containing tubules in Chediak-Higashi lymphocytes. Am J Pathol 69:225-238

25. Craddock PR, Hammerschmidt D, White JG, Salmasso AP, Jacob HS 1977 Complement (C5a)-induced granulocyte aggregation in vitro: a possible mechanism of complement-mediated leukostasis and leukopenia. J Clin Invest 60:260-264

26. White JG 1968 Fine structural alterations induced in platelets by adenosine diphosphate. Blood 31:604-622

27. Karnovsky MJ 1971 Use of ferrocyanide reduced osmium tetroxide in electron microscopy. Am Soc Cell Biol Ann Meeting, New Orleans

28. Graham RC, Karnovsky MJ 1966 The early stages of absorption of injected horseradish peroxidase in the proximal tubules of mouse kidney: ultrastructural cytochemistry by a new technique. J Histochem Cytochem 14:291-302

29. White JG 1971 Interaction of membrane systems in blood platelets. Am J Pathol 66:295-312

30. White JG, Clawson CC 1979 Chediak-Higashi syndrome: spectrum of giant organelles in peripheral blood cells. Henry Ford Hosp Med J 27:286-298

31. White JG, Clawson CC 1980 Chediak-Higashi syndrome Variable reactivity of giant inclusions in polymorphonuclear leukocytes. Ultrastruct Pathol 1:223236

32. White JG, Clawson CC 1979 The Chediak-Higashi syndrome: ring-shaped lysosomes in circulating monocytes. Am J Pathol 96:781 

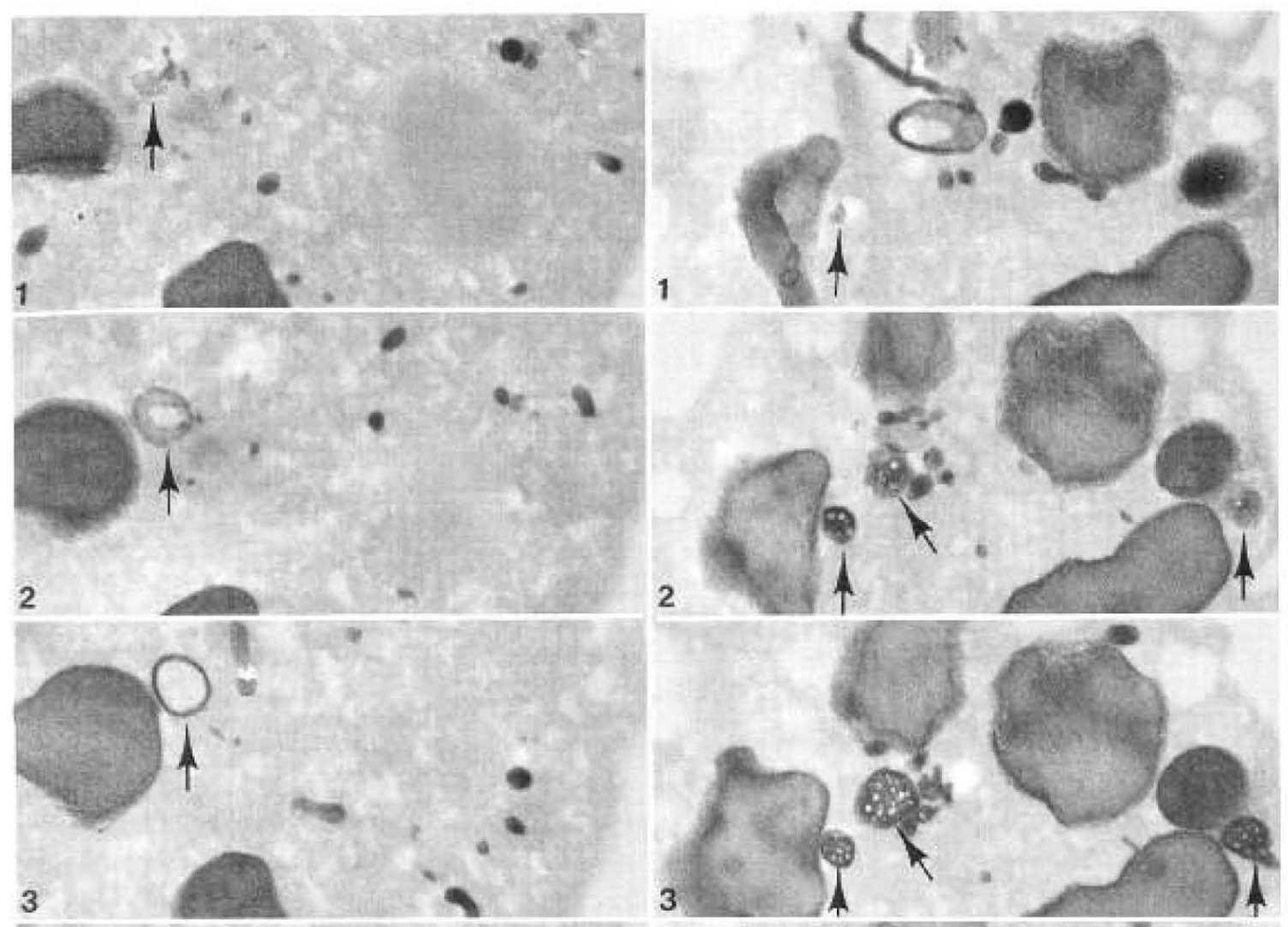

2

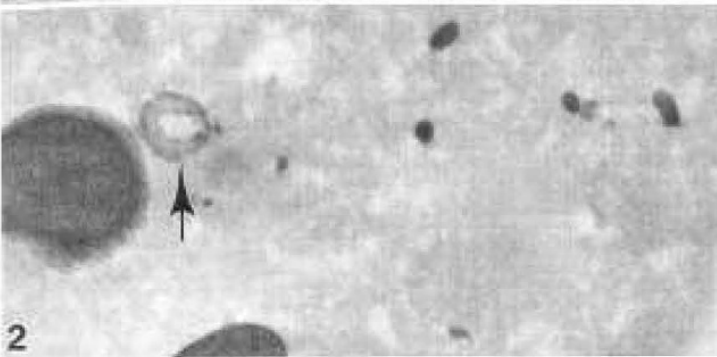

1

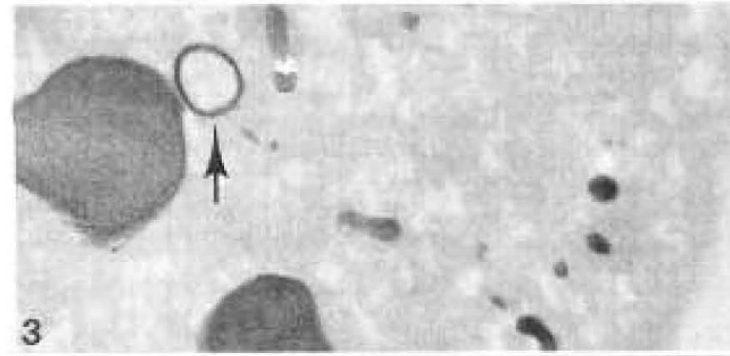

2
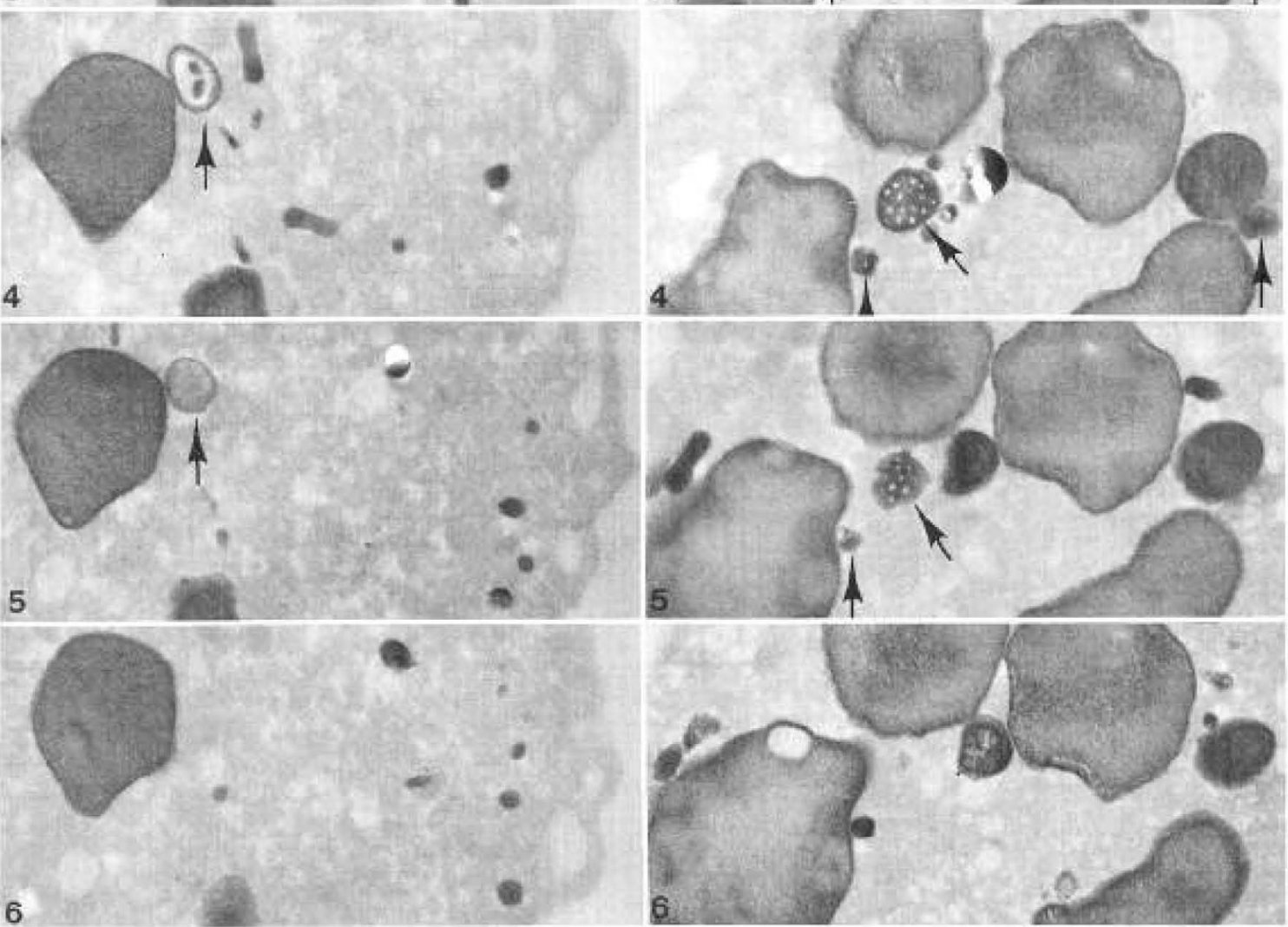

\section{A}

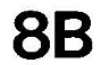

Fig. 8. A, (1-6) serial sections through a ring-shaped lysosome in the cytoplasm of a CHS neutrophil. A tiny fragment $(\uparrow)$ is seen in section 1 . The ring shape is evident in sections 2 and 3 . Two small lysosomes containing reaction product are present within the ring in section 4 . They are gone in section 5 and the ring-shaped lysosome has disappeared in section 6 . Numerous small, normal-sized lysosomes come and go in the serial sections, and a giant organelle remains throughout the sequence. All $\times 16,500$. B, serial sections through honeycomb lysosomes. This form of peroxidase-positive organelle may be unique to CHS neutrophils. A ring type organelle terminates in section 2 and three honeycomb granules $(\uparrow)$ begin. One on the left ends between sections 5 and 6 and is replaced by a dense lysosome. The central honeycomb appears to contact a giant granule in section 5 and is gone in section 6 . The honeycomb on the right disappears between sections 4 and 5 . Small peroxidase-positive granules come and go throughout the sequence. All $\times 16,500$. 

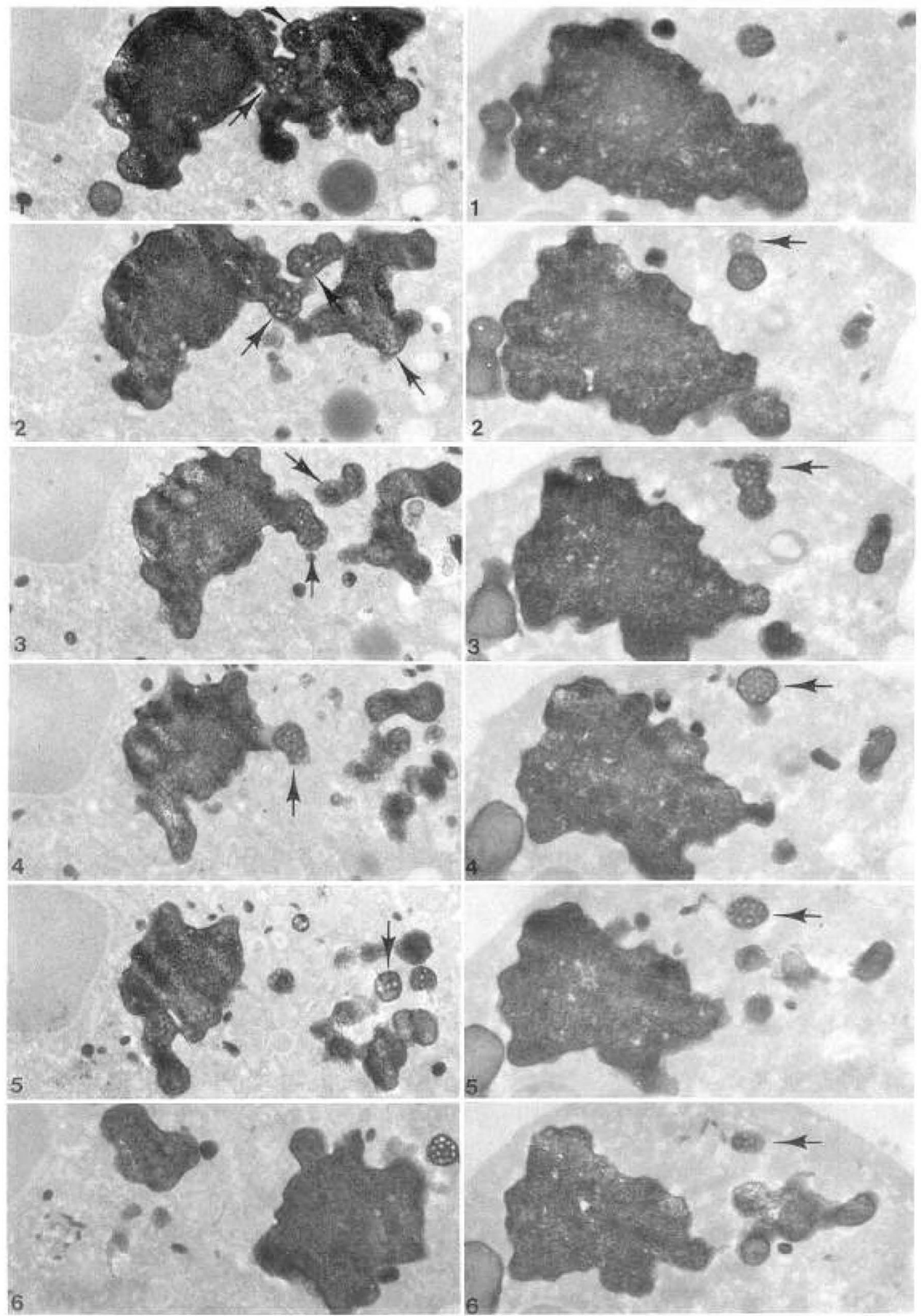

\section{A}

9B

Fig. 9. $A,(1-6)$ serial sections through a complex giant lysosome in CHS neutrophil showing involvement of honeycomb granules in its formation. The huge organelle appears to consist of two parts. The portion on the left separates from the mass on the right and gradually gets smaller. The portion on the right appears made up of honeycomb granules $(\uparrow)$ and one acts as the junction with the lysosome on the left. Individual honeycomb lysosomes become apparent as the mass on the right breaks up into individual units in subsequent sections. In section 5 the mass on the right appears in the process of reforming. In section 6 it has become the dominant structure as the large lysosome on the left disappears. Many small granules positive for peroxidase also come and go in the serial sections. All $\times 14,000$. B, (1-6) serial sections through attached honeycomb lysosomes. The honeycomb granules in section $1\left(_{\uparrow}\right)$ is joined by a second in section $2\left(_{\uparrow}\right)$. The fused organelles are both prominent in section 3 , but the original organelle seen in section 1 disappears in section 4. The remaining honeycomb is nearly gone in section 6 and does disappear in the next section (section 7 now shown) of this series. The development of a fused mass of granules can be followed on the right side of the serial sections. All $\times 16,500$. 